\title{
IMPLEMENTACIÓN DE ATENCIÓN MULTIDISCIPLINARIA DEL PACIENTE CON FIBROSIS QUÍSTICA
}

\section{IMPLEMENTATION OF MULTIDISCIPLINARY CARE FOR PATIENTS WITH CYSTIC FIBROSIS}

\author{
Dr. Héctor H. Gutiérrez \\ Profesor Titular \\ Departamento de Pediatría \\ Universidad de Alabama at Birmingham \\ Birmingham, Alabama, USA
}

\begin{abstract}
IMPLEMENTATION OF MULTIDISCIPLINARY CARE FOR PATIENTS WITH CYSTIC FIBROSIS
Cystic fibrosis (CF) is the most common recessive genetic disease in the Caucasian population. Unanimously, all CF care guidelines in developed countries require that management be provided by a network of specialized CF care centers that cover the entire population of patients, pediatric and adult. The establishment of such centers of excellence requires a level of experience that is achieved only by the conformation of a multidisciplinary team of trained and experienced health professionals, caring for a sufficient number (critical mass) of patients to achieve the best clinical outcomes and survival rates at a lower cost which is achieved by distributing health resources to this small network of centers of excellence. The experience of the health team of the Hospital San Borja Arriaran participating in the project promoted by the COA/UAB CF Center in Birmingham, Alabama in the United States shows that it is possible to transfer knowledge and quality management know-how, creating a cutting-edge, world-class, "CF Center" which performs a multidisciplinary care management of high quality, that is finally reflected in significant improvements in nutritional parameters and lung function of the patients.
\end{abstract}

Keywords: cystic fibrosis; care coordination; health care utilization; multidisciplinary center

\section{RESUMEN}

La fibrosis quística $(F Q)$ es la enfermedad genética recesiva más común en la población caucásica. De manera unánime todas las normativas de cuidado de $F Q$ de países desarrollados requieren que el manejo sea dado por una red de centros especializados de cuidado en FQ que cubra toda la población de pacientes, pediátricos y adultos. El establecimiento de dichos centros de excelencia requiere de un nivel de experiencia que se logra solo por la constitución de un equipo multidisciplinario entrenado y experimentado de profesionales de la salud, a cargo de un número suficiente (masa crítica) de pacientes para lograr los mejores resultados de sobrevida, a un costo menor, lo cual se logra al distribuir los recursos de salud a un número reducido de centros de excelencia. La experiencia del equipo de salud del Hospital San Borja Arriarán en el proyecto impulsado por el COA/UAB CF Center de Birmingham, Alabama de Estados Unidos, demuestra que es posible la transferencia de conocimientos y de gestión de calidad, formando un "Centro FQ" de vanguardia y de categoría mundial, que realiza un manejo multidisciplinario de primera calidad, reflejado en la mejoría nutricional y de función pulmonar.

Palabras clave: fibrosis quística, prestación de atención de salud, centro multidisciplinario

\section{INTRODUCCIÓN}

La fibrosis quística (FQ) es el desorden genético recesivo más común en la población caucásica. El tratamiento de esta enfermedad ha mejorado significativamente en las últimas décadas. Esto ha llevado a una sobrevida más prolongada de los pacientes, especialmente en los países desarrollados, alcanzando ya los 40 años (Figura 1) (1). Desafortunadamente, en Chile ésta aún llega a los 30 años. La causa de muerte por FQ se debe a complicaciones pulmonares en más del $90 \%$ de los casos. Sin embargo, existe gran variabilidad en la severidad y progresión de ésta. Existen diversos factores que influencian la severidad de la enfermedad y la sobrevida. Estos se agrupan en tres categorías: a) genéticos, que incluye aquellos factores biológicos intrínsecos al paciente; b) ambientales, que incluye

\section{Correspondencia:}

Héctor H. Gutiérrez, MD

Division of Pediatric Pulmonary and Sleep Medicine

16007 th Avenue South

Lowder 620

Birmingham, AL 35233; USA

Tel: 1.205.638.9583

Email: hgutierrez@peds.uab.edu factores socioeconómicos, educacionales y demográficos; y c) los relacionados a la prestación de salud, que incluye las intervenciones médicas y la adherencia del paciente a éstas (2). Aunque existen muchas enfermedades cuyo tratamiento es incierto, hay otras tantas condiciones en que aspectos específicos tienen un manejo terapéutico óptimo identificado ya sea por evidencia o por consenso de expertos. En estos casos, la estandarización del cuidado que emana del uso de regímenes apoyados en protocolos científicos y basados en evidencia facilita la prestación de tratamientos de cuidado óptimos y reducen la variabilidad de prestación a prestación, culminando en mejores resultados clínicos (3).

En los últimos 50 años, en los países desarrollados ha evolucionado un sistema de manejo clínico y de seguimiento de los pacientes con $F Q$, constituido por una red de centros especializados de $F Q$ que atienden una masa crítica de pacientes (1-5). Esta red de centros especializados ("Centros FQ") ofrece la mejor manera de organizar equipos multidisciplinarios que intercambian conocimientos, aprenden uno del otro, desarrollan, adaptan y optimizan los diferentes protocolos de tratamiento y resultan en mejor pronóstico y sobrevida (6). Estos centros clínicos de excelencia tienen en común un sistema de manejo clínico altamente desarrollado y óptimamente adaptado a las condiciones locales permitiendo así la aplicación metódica y 
Figura 1. Edad promedio de sobrevida de los pacientes en el Registro Nacional de Fibrosis Quística de la CFF de Estados Unidos desde 1986 a 2013, en incrementos de 5 años

\section{Median Predicted Survival Age, 1986-2013 In 5-Year Increments}

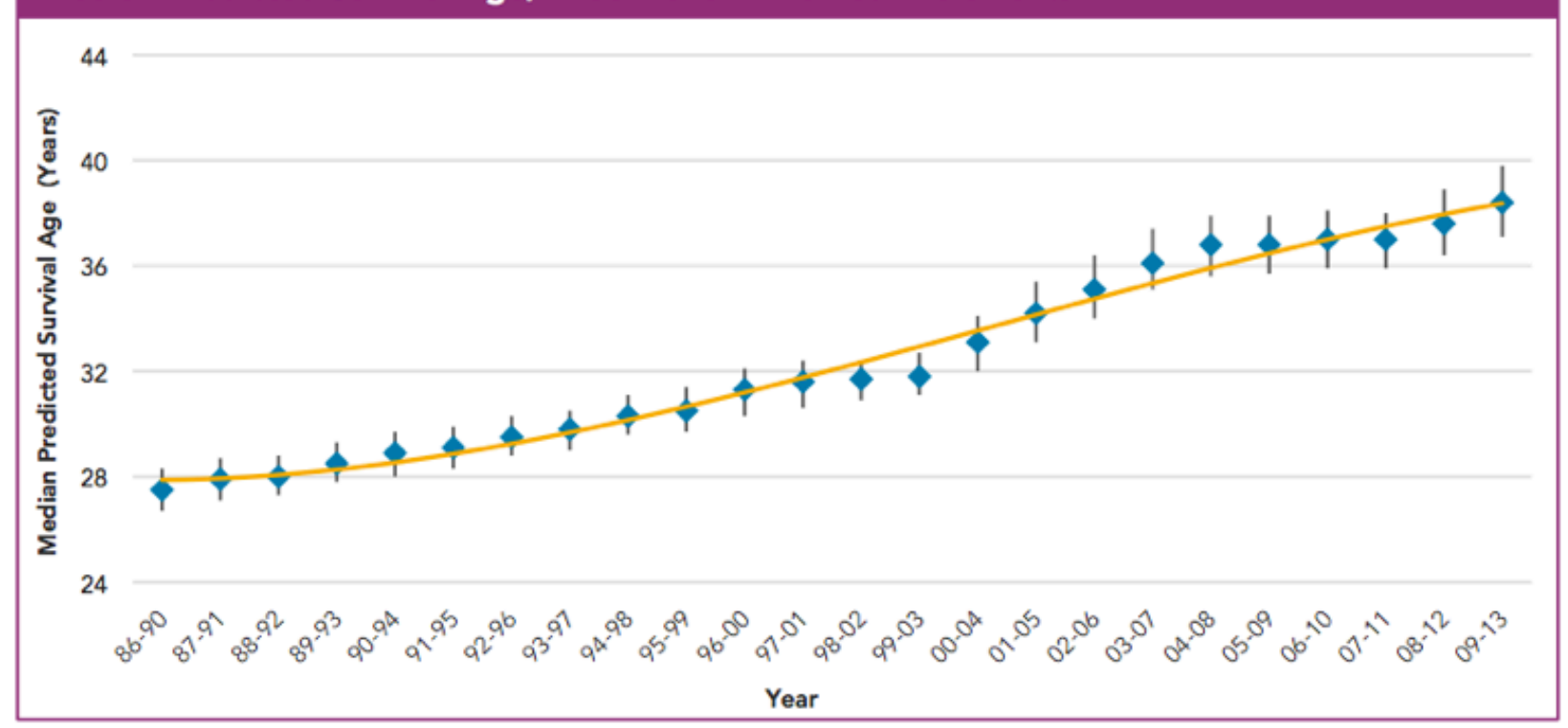

La sobrevida media predictiva para el periodo 2009 - 2013 es 38,4 años (intervalo de confianza 95\%: 37,1 - 39,8 años). Tomado de Cystic Fibrosis Foundation "CFF" Patient Registry; 2013 Annual Data Report to the Center Directors. Bethesda, MD, USA. Usado con permiso de la CFF.

consistente de aquellas terapias y tratamientos basados en la mejor evidencia disponible (7).

\section{MODELO DE CUIDADO EN FIBROSIS QUÍSTICA}

La sobrevida de los pacientes con $F Q$ ha aumentado significativamente como resultado de tratamientos más efectivos, pero especialmente debido a que la mayor parte de los pacientes son atendidos en Centros FQ. Se ha demostrado que pacientes bajo el cuidado de estos Centros FQ tienen mejores resultados clínicos, con mejor nutrición y función pulmonar que aquellos pacientes que no asisten a este tipo de centros (810). Así, el modelo estándar de cuidado óptimo de pacientes con $F Q$ es recibir todo el cuidado que la enfermedad amerita, en el Centro FQ (11-13). Concentrar el cuidado de pacientes pediátricos y adultos con $F Q$ en Centros $F Q$ asegura que el equipo multidisciplinario verá un número suficiente de pacientes para ser capaz de mantener la experiencia en el manejo efectivo, reconocer manifestaciones infrecuentes y demorar el inicio de complicaciones multi-orgánicas asociadas a la enfermedad (1). El número considerado mínimo para que se constituya un Centro $\mathrm{FQ}$ en la mayoría de los países desarrollados es 50 pacientes $(1,2,5,8)$. Además, estos centros conforman una red establecida de cuidado permitiendo al paciente recibir cuidado óptimo en alguno de los centros distribuidos geográficamente.

En el contexto de este modelo de cuidado, los pacientes son evaluados por los diferentes profesionales en cada visita ambulatoria y durante hospitalizaciones. Este cuidado multidisciplinario optimiza el manejo. Además permite una interacción con todos los miembros del equipo del Centro FQ lográndose un manejo comprehensivo, centrado en la familia y paciente, promoviendo una relación de confianza de largo plazo necesaria al requerir $F Q$ un manejo durante toda la vida del paciente.

La implementación y desarrollo de la red de Centros $F Q$ en Estados Unidos ha sido instrumental en el avance de prácticas de cuidado basado en evidencia y la diseminación de nuevo conocimiento de la enfermedad (12).

Las actuales guías clínicas con recomendaciones basadas en evidencia para las prácticas de atención específicas e intervenciones terapéuticas en el cuidado de pacientes con FQ también se esfuerzan por definir las mejores prácticas para la formación de equipos expertos que dan servicios en los Centros FQ. Sin embargo, la adopción y aplicación de estas directrices de atención pueden ser un reto para algunos países de Europa (14) y ciertamente en América Latina y otras regiones del mundo (15) donde los recursos son limitados. Programas terapéuticos deben adaptarse a entornos geográficos, sociales y políticas específicas donde la pobreza, la falta de comprensión del valor de la atención multidisciplinaria y barreras geográficas puede dificultar el acceso al cuidado de FQ. Sin embargo, la centralización del cuidado, organizado en base a Centros FQ y la creación de registros nacionales de datos representan pasos importantes que pueden conducir a una mejora de la supervivencia. Gutiérrez et al (15) sugieren que la clave para mejorar los resultados de FQ en América Latina es mejorando la experiencia y el acceso a la atención especializada de FQ. 
Del mismo modo, Colombo y Littlewood (16) sugieren que los países europeos deben tener a lo menos un Centro $F Q$ con personal adecuado, con mejoras en el financiamiento del cuidado, y existencia de tamizaje neonatal, registro nacionales de pacientes y la formación de organizaciones nacionales de padres y pacientes con $\mathrm{FQ}$.

\section{EL CENTRO DE FIBROSIS QUÍSTICA}

\section{Equipo Especializado en el Manejo de FQ}

Tiene a su cargo un número suficiente de pacientes (mínimo 50) bajo la estructura de un Centro FQ. El nivel de experiencia requerido para tratar $F Q$, una enfermedad compleja, con múltiples sistemas afectados y susceptibles de complicaciones, solo puede ser adquirido por un equipo profesional de la salud multidisciplinario, entrenado, con experiencia y especializado $(1,2,5,8,16)$.

\section{Miembros del Equipo Multidisciplinario $(1,2,5,8)$ Profesionales: \\ 1. Médico especialista (Director Médico) \\ 2. Médico especialista (Sub-Director Médico) \\ 3. Enfermería (Coordinación y Clínica) \\ 4. Asistencia Social \\ 5. Nutrición \\ 6. Kinesiología}

Además, se recomienda apoyo activo de profesionales de Química y Farmacia, Sicología, Terapia Física, y de personal de secretaria.

\section{Tiempo de Dedicación Exclusiva Mínimo Necesario}

La Tabla 1 representa los tiempos de dedicación exclusiva mínimos necesarios para mantener un nivel efectivo de trabajo multidisciplinario por cada profesional del equipo de salud, basado en las recomendaciones de Estados Unidos (8), Europa (6), Australia (5), y del Reino Unido (4), para un centro al cuidado de 50-75 pacientes.

Estos valores pueden variar de acuerdo a la organización de modelo de salud poblacional, factores geográficos y variaciones regionales. Asimismo, los porcentajes deben reflejar el modelo de cuidado que adopta el Centro $F Q$, considerando los tiempos que cada disciplina toma en la evaluación y manejo rutinario, ambulatorio y hospitalizado.

\section{Infraestructura y Servicios}

El centro debe tener acceso a diversos servicios y facilidades para el óptimo manejo de los pacientes. Entre estos: 1. Servicio de hospitalización, incluyendo acceso a unidades de cuidado intensivo, cirugía y otras especialidades.

2. Servicio de cuidado ambulatorio que cumpla con requisitos de prevención de infecciones.

3. Laboratorio diagnóstico, incluyendo:

a. Test del Sudor usando la técnica iontoforesis cuantitativa de pilocarpina (técnica de Gibson \& Cook), siguiendo la guía técnica del Clinical \& Laboratory Standards Institute, de los Estados Unidos (CLSI) (18). b. Pruebas de función pulmonar, incluyendo medición de volúmenes pulmonares.

c. Disponibilidad permanente y continua de:

i. Microbiología

ii. Química de sangre

iii. Hematología

iv. Radiología

4. Servicio de kinesiología respiratoria permanente y continuo.

Asimismo, los pacientes y familias deben tener acceso al Centro FQ para consejo y cuidados de rutina y de emergencia.

Tabla 1. Tiempo dedicación exclusiva de profesionales pertenecientes a Centro de Fibrosis Quística

\begin{tabular}{|l|l|}
\hline Profesional & $\begin{array}{l}\text { Equivalente Dedicación } \\
\text { Exclusiva (44 hrs. = 1.0) }\end{array}$ \\
\hline Director Médico (Médico Cirujano) & 0.5 \\
\hline $\begin{array}{l}\text { Sub-Director Médico } \\
\text { (Médico Cirujano) }\end{array}$ & 0.3 \\
\hline Enfermera Coordinadora & 1.0 \\
\hline Enfermera Clínica & 0.5 \\
\hline Nutricionista & 0.5 \\
\hline Kinesiólogo & 0.75 \\
\hline Otras disciplinas & $0.3-0.4$ (cada uno) \\
\hline
\end{tabular}

\section{Educación Continua y Entrenamiento Adicional}

La educación y el entrenamiento permanente de los miembros del equipo profesional es crítico para el continuo desarrollo de su experiencia en el manejo de pacientes con FQ. Como mínimo, el Centro FQ debe conducir o participar en actividades de entrenamiento y educación tales como: diseminación del manejo de $\mathrm{FQ}$ a los diferentes profesionales de la salud en lo pertinente a sus respectivas disciplinas; participar en la confección de materiales educacionales, guías clínicas de cuidado, colaborar con organizaciones médicas como sociedades, prestadores de salud, gubernamentales, etc.

\section{EXPERIENCIA EN LA FORMACION Y GESTION DE UN CENTRO FQ EN CHILE}

A inicios del 2014, el Centro FQ Pediátrico (COA/UAB CF Center), del Children's Hospital y University of Alabama at Birmingham, en Alabama, Estados Unidos, centro acreditado por la Fundación de Fibrosis Quística de Estados Unidos (CFF, en sus siglas en inglés), y que maneja mas de 300 pacientes menores de 22 años, comienza un proyecto piloto de creación de un Centro FQ en Santiago de Chile, siguiendo los estándares establecidos por la CFF. 
El proyecto tiene como objetivos:

1. Implementar un centro de cuidado de FQ multidisciplinario de vanguardia y de categoría mundial en Santiago de Chile. 2. Entrenar el equipo de salud multidisciplinario del Hospital San Borja Arriarán (HSBA). El entrenamiento incluye visitas periódicas y supervisión continua del equipo de salud de COA/UAB CF Center, tanto en Chile como en Estados Unidos. 3. Mejorar los resultados clínicos nutricionales y de función pulmonar de los pacientes que se controlan por el equipo del HSBA.

En los primeros 18 meses del proyecto, el equipo del HSBA realizó primero una visita de entrenamiento en Birmingham donde recibe extensa exposición y educación en el manejo ambulatorio y hospitalizado de pacientes con $F Q$. Se pone especial énfasis en trabajo multidisciplinario, uso de protocolos de tratamiento, adquisición de datos para evaluar progreso clínico, herramientas de laboratorio, y revisión critica de las Guías Clínicas de Cuidado del Paciente con FQ de la CFF. En cuatro visitas subsecuentes del equipo de COA/UAB a Santiago, se realiza una evaluación en profundidad y en conjunto de la totalidad de los pacientes que maneja el equipo del HSBA. Se hacen notar las diferencias en el manejo de los pacientes locales, se destacan los aspectos positivos y se mejoran aquellos deficitarios.

\section{Cambios en el manejo nutricional}

El manejo nutricional en el HSBA antes de comenzar el entrenamiento se caracterizaba por: a) todos los pacientespancreáticos insuficientes estaban recibiendo menos de $50 \%$ de la dosis recomendada de enzimas pancreáticas. La mayoría de ellos aún presentaban signos y síntomas de malabsorción; b) ajuste por parte de los padres a una dieta baja en grasa debido a que al ingerir más de ellas, los síntomas de malabsorción empeoraban; c) el esquema de administración de enzimas era rígido y complicado; d) creencia generalizada por parte de los padres de que el aumentar la dosificación de enzimas significaba que había habido un empeoramiento de la enfermedad; e) tiempo insuficiente para educar a las familias sobre temas nutricionales, con ideas erróneas generalizadas sobre la nutrición y FQ; f) uso infrecuente de suplementos nutricionales y de recomendación de gastrostomía en aquellos pacientes que lo ameritaban en base a las guías clínicas de la CFF; g) poco énfasis en el autocuidado del paciente y de empoderamiento de los padres en el cuidado de sus hijos.

Varios aspectos se corrigieron. Entre estos: a) entrenamiento intensivo de la nutricionista en anamnesis nutricional, aspectos de autocuidado del paciente, reconocimiento de cambios clínicos sutiles que requerían intervención, seguimiento enfocado a la mejoría del estado nutricional, etc.; b) aumento de dosis de enzimas pancreáticas a la totalidad de los pacientes (62\% de aumento promedio, con un rango de $10 \%$ a $117 \%)$; c) aumento de ingesta calórica en un $40 \%$ promedio; d) educación nutricional, eliminación de inexactitudes sobre el significado en aumentar la dosificación de enzimas pancreáticas; d) simplificación de la administración de enzimas pancreáticas.

Esta mejora significativa en los resultados nutricionales se logró en un período relativamente corto de tiempo. El consenso general de los dos equipos multidisciplinarios fue que tutoría intensiva, en terreno, práctica, y con retroalimentación periódica fue muy eficaz en la transformación de la lógica de las intervenciones nutricionales y muchos aspectos de educación familiar (Figura 2).

Figura 2. Cambios en el índice de masa corporal (IMC), Hospital San Borja - Arriarán

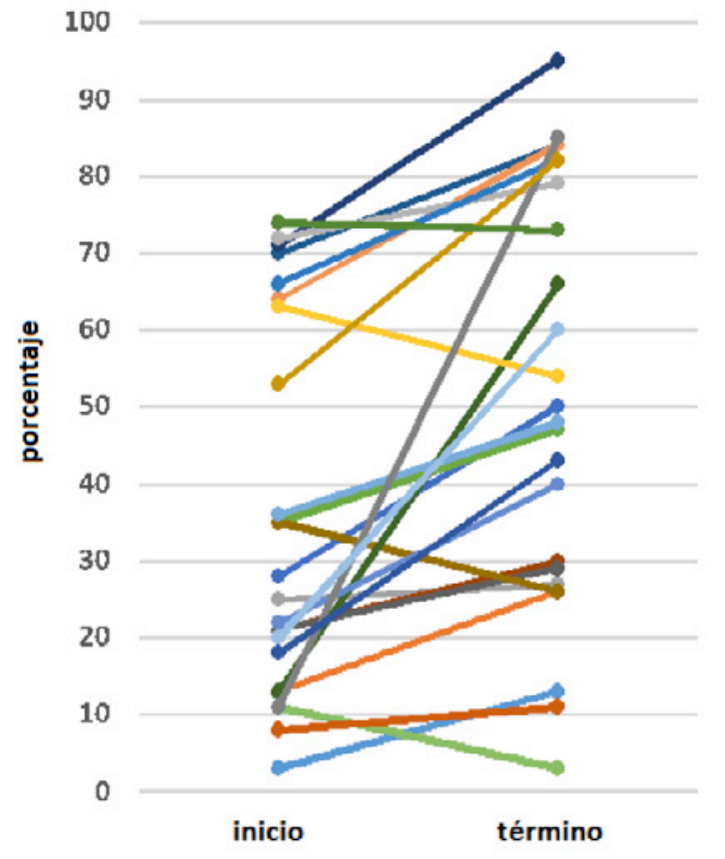

Cambios en el índice de masa corporal (IMC) de 25 de 38 pacientes evaluados en el Centro FQ del HSBA en un periodo de 18 meses. En promedio el IMC subió del $28 \%$ al $50 \%$ en la población bajo cuidado del HSBA.

\section{Cambios en el Manejo Respiratorio}

El manejo respiratorio en el HSBA antes de comenzar el entrenamiento se caracterizaba por: a) poco énfasis en la importancia de la fisioterapia respiratoria; b) tiempo insuficiente para educar a las familias sobre temas respiratorios y $F Q ; c)$ dependencia casi exclusiva de kinesiterapia respiratoria a ser realizada por profesionales; d) falta de entrenamiento a los padres sobre la realización de kinesiterapia respiratoria; e) evaluación irregular de la función pulmonar.

Una vez que se inicia el trabajo en conjunto, se introducen cambios significativos, reflejando el modelo y estilo de atención dispensada en el Centro FQ de Birmingham. Entre estos: a) aumento del tiempo para la educación y la reevaluación de las intervenciones de salud pulmonar en cada visita clínica; b) enseñanza de las técnicas de kinesiterapia respiratoria a realizar por los cuidadores; c) énfasis en realizar kinesiterapia respiratoria en el hogar, por los padres, en lugar de recibirla en el policlínico, realizada por un profesional; d) re-evaluación y reforzamiento de la técnica de kinesiterapia respiratoria en cada visita ambulatoria; e) empoderamiento de los cuidadores para 
realizar kinesiterapia respiratoria durante las hospitalizaciones de su hijo.

Los cambios implementados llevan a una mejora significativa en la función pulmonar en un período relativamente corto de tiempo. El porcentaje del volumen expiratorio forzado del primer segundo (VEF1\%) mejoró en mas de 10 puntos porcentuales. El traspaso de la realización de kinesiterapia respiratoria a los padres fue el cambio más significativo y presentó un alto grado de satisfacción por parte de los padres y del equipo de salud.

\section{CONCLUSIONES}

Para lograr los mejores resultados clínicos en el manejo de pacientes con fibrosis quística es necesario que estos sean vistos por equipos multidisciplinarios especializados y con gran experiencia viendo un número significativo de pacientes. Estos equipos se deben conformar como Centros de Fibrosis Quística de excelencia y conformar una red especializada de atención a lo largo del país. Los centros tienen la responsabilidad y desafío de buscar las mejores terapias y modalidades de tratamiento basado en evidencias, perfeccionarse de manera continua, compartir información y diseminar públicamente sus resultados clínicos. La red de Centros FQ de Estados Unidos y de otros países desarrollados es el modelo a seguir, adaptados a la realidad local. Chile talvez necesita de una media docena de estos centros distribuidos a los largo del territorio. La experiencia piloto mostrada de manera breve en esta comunicación, demuestra que es posible la transferencia de gestión de calidad y de mejores prácticas, replicando entonces lo que constituye un Centro FQ.

\section{El autor declara no presentar conflicto de intereses}

\section{REFERENCIAS}

1. Cystic Fibrosis Foundation Patient Registry. Annual Data Report to the Center Directors. Bethesda, MD, USA; 2013

2. Wolfenden LL, Schechter MS. Genetic and non-genetic determinants of outcomes in cystic fibrosis. Paediatr Respir Rev 2009; 10: 32-36

3. Horbar JD, Rogowski J, Plsek PE, Delmore P, Edwards WH, Hocker J, Kantak AD, Lewallen P, Lewis W, Lewit E, McCarroll CJ, Mujsce D, Payne NR, Shiono P, Soll RF, Leahy $\mathrm{K}$, Carpenter $\mathrm{JH}$. Collaborative quality improvement for neonatal intensive care. NIC/Q Project Investigators of the Vermont Oxford Network. Pediatr 2001; 107: 14-22

4. Standards for the clinical care of children and adults with cystic fibrosis in the UK. London: UK Cystic Fibrosis Trust; 2011

5. Bell SC, Robinson PJ. Cystic fibrosis standards of care Australia. In: Fitzgerald DS, editor. Sydney, NSW: Cystic Fibrosis Australia; 2008

6. Clinical Practice Guidelines for Cystic Fibrosis. Cystic Fibrosis Foundation. Bethesda, Maryland, USA; 1997

7. Conway S, Balfour-Lynn IM, De Rijcke K, Dřevínek P, Foweraker $J$, Havermans $T$ et al. European Cystic Fibrosis Society
Standards of Care: Framework for the Cystic Fibrosis Centre. J Cyst Fibros : Official Journal of the European Cystic Fibrosis Society 2014; 13 Suppl 1, S3-22

8. Smyth AR, Bell SC, Bojcin S, Bryon M, Duff A, Flume P et al. (2014). European Cystic Fibrosis Society Standards of Care: Best Practice guidelines. J Cyst Fibros : Official Journal of the European Cystic Fibrosis Society 2014;13 Suppl 1: S23-42

9. Johnson C, Butler SM, Konstan MW, Morgan W, Wohl MEB. Factors influencing outcomes in cystic fibrosis: a centerbased analysis. Chest, 2003;123: 20-27

10. Criteria for center and program accreditation. Cystic Fibrosis Foundation, Bethesda, MD, USA; 2015.

11. Mahadeva R, Webb K, Westerbeek RC, Carroll NR, Dodd ME, Bilton D. Clinical outcome in relation to care in Centres specialising in cystic fibrosis: cross sectional study. BMJ 1998;316:1771-1775.

12. Johnson C, Butler SM, Konstan MW, Morgan W, Wohl ME. Factors influencing outcomes in cystic fibrosis. A centerbased analysis. Chest 2003;123:20-27

13. Gutierrez HH, Mogayzel PJ. ESCF care guidelines beyond Europe. J Cystic Fibros 2014;13, 359-360

14. Mogayzel P J, Dunitz, J, Marrow L C, Hazle L A. Improving chronic care delivery and outcomes: the impact of the cystic fibrosis Care Center Network. BMJ Qual Saf 2014;23 Suppl 1: i3-i8

15. Gutierrez, H H, Sanchez I, Schidlow DV. Cystic fibrosis care in Chile. Curr Opin Pulmon Med 2009;15: 632-637

16. Colombo C, Littlewood J. The implementation of standards of care in Europe: state of the art. J Cyst Fibros 2011;10(Suppl. 2):S7-S15

17. Schechter MS, Gutierrez HH. Improving the quality of care for patients with cystic fibrosis. Curr Opin Pediatr 2010; 22: 296-301

18. CLSI. Sweat Testing: Sample Collection and Quantitative Chloride Analysis; Approved Guideline-Third Edition. CLSI document C34-A3. Wayne, PA: Clinical and Laboratory Standards Institute; 2009 